\title{
Advanced Photonic and Electronic Systems WILGA 2017
}

\author{
Ryszard S. Romaniuk
}

\begin{abstract}
WILGA annual symposium on advanced photonic and electronic systems has been organized by young scientist for young scientists since two decades. It traditionally gathers more than 350 young researchers and their tutors. Ph.D students and graduates present their recent achievements during well attended oral sessions. Wilga is a very good digest of Ph.D. works carried out at technical universities in electronics and photonics, as well as information sciences throughout Poland and some neighboring countries. Publishing patronage over Wilga keep Elektronika technical journal by SEP, IJET by PAN and Proceedings of SPIE. The latter world editorial series publishes annually more than 200 papers from Wilga. Wilga 2017 was the XL edition of this meeting. The following topical tracks were distinguished: photonics, electronics, information technologies and system research. The article is a digest of some chosen works presented during Wilga 2017 symposium. WILGA 2017 works were published in Proc. SPIE vol.10445.
\end{abstract}

Keywords: photonic systems, electron technology, material engineering, electronics, photonics, optoelectronics, lasers, telecommunications, informatics, electronic systems, large research experiments, young researchers meetings, WILGA Symposium, Wilga

\section{INTRODUCTION}

$\mathbf{T}$ HIS year, the Symposium of Young Scientists WILGA Photonics Applications and Web Engineering marked a modest, yet round, $40^{\text {th }}$ edition and $20^{\text {th }}$ anniversary of its diligent service to the photonics and electronics research communities, primary of young scientists. The symposium gathered nearly 400 participants, young scientists from all over the country and from abroad. There were presented over 350 research papers, out of which, after peer review, around 250 were published in Proc. SPIE. vol.10445. Wilga Symposium enjoys a patronage of the Committee of Electronics and Telecommunications of the Polish Academy of Science [1], Polish Optoelectronics Committee of SEP, Photonics Society of Poland [2-3], IJET Journal [4], SPIE - The International Society for Optics and Photonics [5-10], and WEiTI WUT. Wilga Symposium works are published in Proc.SPIE, Photonics Letters of Poland, IJET, Elektronika. Wilga Symposium has always several sessions on large research experiments including high energy particle physics, astroparticle physics, hot plasma fusion energy, free electron lasers and large accelerator infrastructures [11-18], as well as sessions on optical fibres and photonics [19-21]. The participants come from major technical universities around the country like WUT Warsaw, BUT Białystok, LUT - Lublin, GUT Gdańsk, WrUT Wrocław, KiUT Kielce, KrUT - Kraków, SUT - Silesian, etc. Usually there is also a number of young researcher participants from abroad. Wilga is an efficient mixer of national young science in photonics and electronics showing the current advances in the work and in the research interests of young people.

The following main topical tracks were organized during the XL WILGA 2017 Symposium:

- Photonics: optical fibre communications, bio-photonics and opto-genetics, optical and optoelectronic sensors and photonic systems components, application of photonic equipment in research, astronomy, and industry;

- Electronics: functional systems development, applications in research and industry, functional IoT systems;

Informatics: information technologies, software engineering, hardware-software co-design, artificial intelligence development for functional systems, bioinformatics;

- System research: theory, design, construction and implementation of large and very large functional systems;

- Applications of complex functional systems in the following sectors: safety and defence, airborne and space, large research experiments, large functional infrastructures, energy and large industrial utilities.

Below we show a short digest of chosen work presented during XL Wilga 2017 Symposium.

\section{MATERIAL ENGINEERING AND NOVEL MATERIALS APPLICATIONS}

Nano materials are used for textronic technologies. Textronics is a knowledge and technology of new textile materials to be used for making of new generation of clothing. Textile fibres are coated with carbon nanotubes for smart clothing applications. Smart clothing technology embraced numerable solutions starting with simple ones containing a confined number of sensors, going to very complex ones coupled with body sensor network BSN. There are tested, at Mechatronics WUT, covering methods with nanotubes of several textile fibres including cotton, silk, wool, polyester and polyamide. CNT material preparation used CNT carpets, acetone and ultrasonic methods for homogenization and cleaning. Test coating used immersion, spray and painting, with spray as the best choice. Silver interconnects were printed on foil using different structures: single, double and multi-layered with CNT layer. The foil was integrated with textiles. Sheet resistance was measured after printing and after laundry. Multi-layered structures were the best choice due to smaller fragility. CNT were integrated into PAN polyacrylonitrile composites for flexible textronics and the properties of the material were investigated.

Various novel nanomaterials, including carbon allotropes like carbon nanotubes, are extensively tested for biomedical applications. Applications of the CNT in biomedicine include: 
mechanical actuators for artificial muscles, biosensors for structural health monitoring, electrodes for deep brain stimulation and treating of movement disorders, tissue engineering for cells differentiation, migration and growth. One of the issues combined with biomedical applications of the CNT is increasing their conductivity and relevant coating against biological environment. A conductive polymer paste was designed with nano-silver particles. The main requirement is biocompatibility. Several technologies were tested and compared, including painting, flooding and dipping. Spin and spray coating are considered. CNT fibres were also p-doped with F4TCNQ for research on conductivity.

Transparent electrodes are needed for many practical applications of electronic components, assemblies and devices. Ultrasonic spray coating technique was used for manufacturing of transparent high power electrodes for flexible heaters. Large area transparent heaters are widely used in goggles, photovoltaic cells working at high altitudes, car windshields, etc. There are used most commonly the following TCF materials for transparent electrodes: ITO, ZnO, FTO and ATO. ITO disadvantages are: brittle, vacuum technology required, and has only semiconducting properties. Metal nanoparticles, graphene, transparent organic conductors, CNTs and other metal oxides are researched for optimal TCF solutions. Ultrasonic atomisation of TCF materials results in smaller overspray and material loss than in pure pneumatic atomisation. Substrate plotter powered by ultrasonic generator is equipped in ultrasonic head supplied with liquid TCF material suspension. Solvents for CNTs suspensions are: acetone, nitromethane, ethanol, EGEA, methyl di-glycol, and water. Overlay efficiency and thin film parameters, like thickness and roughness, depend on suspension viscosity and flow, and ultrasound power. TCF parameters were measured at Mechatronics WUT including characteristic surface resistance and mechanical parameters including fatigue and strength of adhesion. Heater demonstrator was manufactured and tested.

Aerosol jet printing AJP was compared to spray coating SC. AJP is suitable for pattern printing, with linewidth about $10 \mu \mathrm{m}$, is suitable for substrates of complex shape, and has to be equipped with nozzle clogging prevention device. AJP is confined by high ink rheology, requires complicated and expensive machine, and printing process is slow what results in long printing time for big patterns. SC is suitable for big area coating and substrates of complex shape. The equipment is easy, simple and cheap, and the printing process is fast. SC drawbacks are: easy nozzle clogging, big overspray effect what makes it unsuitable for small patterns and lines. The aim of comparison is to find optimal technology for printing of conductive nanosilver lines.

Novel materials for die attachment by LTJT are researched containing silver nanoflakes of submicron size covered with nano silver grains. Nano-pastes with silver nanoparticles are researched for bonding purposes. Such pastes have low sintering temperatures, around $250^{\circ} \mathrm{C}$, due to high surface energy, high electrical and thermal conductivities, exhibit no glass frit, are cheaper than gold and platinum, and meet recent RoHS requirements. The following feature of mixed micro and nanosilver pastes are beneficial: lower paste viscosity, easier deposition and better levelling, less organic substances, reduced gas bubbling disturbing die bonds, easier gas escape, microflakes provide better adhesion to flat surfaces and better die metallization. The mixed silver pastes were proven to give better bonding properties than classical solders and adhesives.

$\mathrm{pH}$ sensitive layers were researched, based on graphene and ruthenium dioxide, for potentiometric sensors and biomedical applications like smart bandages modified with enzymes, ions detection in sweat for muscle activity monitoring, etc. Several types of pastes were tested for this purpose and their sensitivities to UV irradiation. The research aims at construction of complex devices to be applied for body network sensors.

Lab on paper concerns smart functional devices, sensors, microfluidic channels carved out directly by laser or other methods in a kind of porous chemical paper. Impregnation parts of such a structure with proper chemistry makes out of this region a sensor. Concatenation of such sensors along the microfluidic track makes a network of sensors, all carved in a sheet of disposable paper or in a foil. Imposing one structure over the next ones makes 3D sensing volume. Research on integrated LoP/LoF devices is done at ISE WUT. There were integrated several sensors in a single circuit containing tiny colorimeter, $\mathrm{pH}$ meter and salinity meter.

Carbon nanotubes CNT films deposited on different silica substrates were studied by Raman spectroscopy and scanning electron microscopy (ITR, KiUT). The film samples were prepared by two steps methods consisting of PVD and CVD processes. Ni-C nanocomposite films were obtained by evaporation on porous silica and DLC/porous silica substrates. The type of silicon substrate significantly influences the structure of the analysed layers. In particular, graphitization of carbon matrix was observed depending on the pore size. DLC layer presence had no significant effect on the shape of Raman spectra.

Standard and hybrid solar cells were sensitized by various dyes at SUT in Gliwice. Conductive polymers were applied for fabrication of organic photovoltaic cells. Apart of OPC these materials are used for infrared and radar absorbing films, LEDs, OLEDs, antistatic layers, and various sensors including gas sensors. Organic photovoltaic structures can be single or double layered or heterojunction doped with donor and acceptor. Hybrid solar cells have additionally hole and electron transport layers for separating LUMO and HOMO energy layers, and glass substrate, and can be of normal and inverted geometry. $\mathrm{TiO}_{2}$ or anthocyanin dye-sensitized OPC contains organic dye that acts as an electron donor and an inorganic semiconductor that accepts electron acceptor and transfers them to the electrode. The advantages are: low cost, nontoxic, biocompatible, good absorption in UV and blue part of spectrum.

\section{Photonic Components, SEnsors AND SENSOR Systems}

Chirped and tapered fibre Bragg grating based Fabry-Perot BGF cavity was designed and fabricated at ISE WUT. This lab specializes in FBGs. Such BGFs were manufactured using two uniform phase masks with precisely defined period difference. Other method was with single phase mask with linearly changing chirp and with adjusted tapered fibre slope. The FP cavity was created between two FBRs located along the fibre cone. Matching condition for TCFBG was obtained. Detuning characteristics were measured for different cavity lengths versus taper profile slope and phase mask chirp rates. There was observed the influence of the initial strain on the Bragg 
wavelengths detuning of the FP cavity written on tapered fibre with different slopes. Positioning of the fibre behind the phase mask leads to some initial strain during grating inscription. Reduction of the fibre diameter leads to mode field expansion and reduction of the grating reflectivity written in tapered fibre of smaller radius. Taking into account these effects allows to calculate complex FBG spectral response. Strain and temperature changes were applied to these FBG constructions.

Temperature tracking system in special harsh environment constraints was designed and constructed basing an FBG and Peltier module. Photonic solution measures low and high temperatures, is immune to EM radiation and exhibits chemical inertness. The compact sensing circuit consisted of two FBGs, one measuring and the second sandwiched between two Peltiers. Other version of the system, basing on SFP modules for multiparameter measurements is designed for IoT applications.

Sensor research team at ISE works on different solutions of microsystems with numerable sensor constructions including: heart rate, skin impedance, some chemical and biochemical parameters, sweat $\mathrm{pH}$ and salinity, water loss through skin, subminiature interdigital humidity sensors with heaters, etc. Some of these microsystems are integrated with clothing/garment or other wearable devices and practically tested. Different grades of MEMS accelerometers were tested for error characteristics. Some of these sensors used in all smartphones are said to have big measurement errors. The errors are: misalignment, temperature related, scale factor, cross-coupling and fixed biases. Stochastic errors of MEMS accelerometers are associated with measurement noise, random walk error and turn on to turn off/on biases. Three axial, wide range economic and advanced accelerometers were compared. Test platform was a precisely scaled, high resolution centrifuge powered with stepper motor. Tests have revealed that low grade accelerometers are highly nonlinear and no simple calibration method can solve the issue. Different quality of $\mathrm{z}$ axis was observed. Device parameters differ considerably for individual specimen. Work on MEMS accelerometers is also done at BUT, with the purpose to implement a complementary filter for the precise estimation of the angle of rotation around a chosen axis of the examined object. There is presented an example of designing and implementing digital filters based on analogue prototypes.

Advanced sensor system was researched for shale gas exploration and production. Hydraulic fracturing requires injection into the rock water, sand and chemicals at high pressure down and across into horizontally drilled wells $3 \mathrm{~km}$ below the surface. The pressurized mixture causes the rock layer, which is Marcellus Shale, to crack. These fissures are held open by the sand particles so that natural gas from the shale can flow up the well. One of the employed measurement methods used magnetic hysteresis to build 3-axis fluxgate magnetometer. A full, autonomous, battery operated downhole system was designed and tested in real life conditions. One of the system components was detection of groundwater contamination in direct vicinity of shale gas well. The hydraulic fracturing liquid is complex and contains several components for different purposes. Diluted acid is used to dissolve minerals and initiate cracks in the rock. Biocide components eliminates bacteria in the water that produce corrosive by-products. Breaker allows a delayed break down of the gel polymer chains. Corrosion inhibitor prevents the corrosion of the pipe. Crosslinker maintains fluid viscosity as temperature increases. Friction reducer minimizes friction between the fluid and the pipe. Gel thickens the water in order to suspend the sand. Iron control prevents precipitation of metal oxides. Salts create a brine carrier fluid. Oxygen scavenger removes oxygen from the water to protect the pipe from corrosion. $\mathrm{pH}$ adjusting agent maintains the effectiveness of other components such as crosslinkers. Proppant allows the fractures to remain open so the gas can escape. Scale inhibitor prevents scale deposits in the pipe. Surfactant is used to increase the viscosity of the fracture fluid. The following water parameters were measured by the water pollution alarm system: conductivity, temperature, salinity, total dissolved solids, water density, water level, $\mathrm{pH}$, chloride contents, and some other ones. Multi-parameter, down-well, ruggedized sensing head was supplemented with on-surface, GSM networked full weather station. The system was successfully deployed with no major failure observed. The analysed groundwater in the vicinity of the drilling field was classified as good quality water.

Dew point hygrometer was researched using capacitance based interdigital structures and manufactured by multilayer ink jet printing (ISE WUT). Fully functional dew point hygrometer was shown with stable capacitance characteristic. New fabrication process for multilayer ink jet printed structure shows some advantages over classical fabrication methods.

Very small mili-Ohm resistance measurement systems require special attention as they are used in many practical sensory solutions. Designing optimal sensor and their controller results in reduction of transient time in temperature measurements. Work on industrial class measuring multisensory systems are done at BUT.

Compensation of hard- and soft-iron distortions in magnetometer measurement data is researched at BUT. Tilt compensation method was chosen on data with ferromagnetic influence. Calibration procedure was proposed, including yaw angle estimation and calculation of compensating coefficients. Magnetometer measurements depend on their orientation thus it is necessary to use additional compensation of their tilts. Calculation of compensations is done numerically.

Intelligent buildings are equipped in increasingly complex sensory networks and working environment management systems for interior spaces. The factors impacting the working conditions of humans include: air temperature to provide thermal comfort, relative humidity, carbon dioxide, carbon monoxide, other chemical pollutants, flying dust concentration, air pressure, lighting, heating, ventilation, audio noise, level of sound, overall microclimate, etc. Smart buildings should provide user comfort with energy savings. A multi sensor test system was realized at BUT and TU Ostrava using several listed sensors and ARM Cortex microcontroller.

Sensor head based on nanomaterials was manufactured and characterized for hydrogen detection. C-Ni films deposited on different substrates are researched for applications as field emitters and sensors. Carbonaceous films containing Ni/Pd are used for gaseous $\mathrm{H}$ detection. The films are deposited by PVD method in ITR. $\mathrm{C}_{60}$, nickel and palladium acetates are evaporated from separate sources at dynamic vacuum. Vacuum evaporation method assisted by the electron beam was chosen to produce contacts and with the use of materials of high temperature of melting. The structure retains good bondability, and electric and adhesive properties of titanium. Contacts with 
wire bonding are used to fabricate hydrogen sensor. C-Pd films with lower Pd content have carbonaceous matrix composed of amorphous carbon, fullerene grains, residues of partial decomposition of fullerene and acetate molecules. Palladium is in a form of fine grains because there are no Pd peaks in the $\mathrm{X}$ ray spectrum. Residues of organic particles originating from palladium acetate are observed in FTIR spectra. C-Pd films with higher content of palladium has a form of $\mathrm{Pd}$ metallic nanograins with sizes bigger than a few nanometres. The grains are positioned in carbonaceous amorphous matrix. C-Pd films are stable up to approximately $200^{\circ} \mathrm{C}$, and above this temperature start to decompose. The Pd decoration on CNTs, made by combination of PVD and CVD methods, are creating depletion regions which enhance the sensor response by control of carrier mobility and density.

\section{OPTICAL COMMUNICATIONS AND COMPUTER NETWORKS}

Optical communication session was organized by ITele PW optical communications laboratory. One of the key issues in complex optical networks with multilevel PAM signals is monitoring of the transmission quality. Monitoring should be done in an asynchronous way, not to interfere with the regular production transmission of the system. Signals transmitted in hybrid optical networks are of different rate and different protocols. There are tested phase diagram methods to assess the transmission quality. These methods are used in parallel to the ultimate measure of the Quality of Service which is BER. BER measurement involves full synchronous status and signal decoding, which is complicated, costs a lot, requires long time, and if time is too short, the result is often irrelevant. Alternatively $\mathrm{Q}$ parameter is measured, with exact $\mathrm{Q}$ relation to BER for Gaussian noise. MER and EVM measures are used for QAM modulations with no necessity of signal decoding. These methods are of high cost for high bit rate signals. Asynchronous signal sampling omits the above cost and complication issues of the QoS estimation. Sampling provides a histogram from which the Q parameter is calculated. The next calculation task is to combine this asynchronous Q parameter with the QoS. The difficulty is that the phase diagrams for multilevel signals are much more complicated than these ones for binary signals.

Asynchronous sampling method can be used for optical transmission testing also by image analysis containing chromatic dispersion. Different analytic methods are used including convolutional neural networks. Optical fibre digital signal is subject to several impairments in physical layer like chromatic dispersion, polarization mode dispersion and ASE noise. Transmitted signal is deteriorating along the fibre length, attenuated and dispersed. Testing system consists of tuneable $1550 \mathrm{~nm}$ laser, MZ modulator with sampling input at $10 \mathrm{Gbps}$, SMF, EDFA band pass filter, photodetector, splitter with electrical delay by $1 / 4,1 / 2$ and 1 bit, and output phase portrait displayed for all kinds of signal impairments separately and combined. The phase portraits, treated as images, are subject to data analysis done for example by deep learning and CNN method. CNN are well suited for analysis of multi-dimensional input signals. A lot of measurement data is required to give precise results. Then, the estimation of signal impairments are sufficiently good.

CAP carrier-less amplitude-phase and PAM - pulseamplitude modulation robustness in optical links are compared against mode partition noise present in multimode VCSEL semiconductor lasers. Most of the traffic is generated inside the data centres. Usage of cheap MMF solutions there for local interconnects is combined with several confinements like: limited component bandwidth, modal and chromatic dispersion, laser relative intensity noise and mode partition noise. Mode partition noise stems from power sharing among many modes, mutual competition among modes, power variation in individual modes, and chromatic dispersion, which results in random pulse temporal jitter at the fibre output. Simulations were performed for $25 \mathrm{Gbps}$ signals and non-equalized and equalized modulations with VCSEL, and $100 \mathrm{~m}$ MMF. PAM revealed to be more robust than CAP in these conditions.

Optical fibre transmission costs can be lowered considerably by using low cost components and multimode fibre option. A confined multimode bandwidth may be widened by using modal filter narrowing the spectrum of transmitted modes to the lowest ones. VCSEL sources are used for such low cost option. High order modal filter introduces some excess losses which depends on modal number. The filter may also improve the modal profile in the fibre. High order mode filter inserted in optical path improves the quality of multimode data transmission and is a low cost solution. The drawbacks are high insertion losses which may be compensated by amplification and some additional modal instability.

Optical fibre link design and optimization includes fitting the dispersion characteristics of the used transmission window to the required bit rate. The used bit rates are 0,4 and 1,0 Tbit/s. Such optimization concerns the Ethernet physical layer design, especially in the data centres where the data rates grow unstoppably. Cost-effective and energy efficient high speed solutions are needed. Very short links, up to a few hundred $\mathrm{m}$ are serviced well by 850 SVCEL and MMF. $1550 \mathrm{~nm}$ systems are not necessarily cost effective in not really trunk applications. $1310 \mathrm{~nm}$ systems offer yet unutilized potential where wide base components are available and still a limited chromatic dispersion penalty is involved. $1550 \mathrm{~nm}$ window offers even 10Tbps capacity while $1310 \mathrm{~nm}$ widow may carry signals up to hundreds Gbps. The assumptions for $1310 \mathrm{~nm}$ systems are: all semiconductor, at least $40 \mathrm{~km}$ distance, system bit rate at least $400 \mathrm{Gbps}$ with line rates 40 or $50 \mathrm{Gbps}$. OOK modulation is used for 50 Gbps. PAM, CAP, QPSK modulations are used for over $100 \mathrm{Gbps}$. The following system features are compared: energy, latency, footprint, FEC and cost. The system consists of: data input, LD, EAM, Mux, $40 \mathrm{~km}$ SSMF, SOA, Demux, PR and data output. Numerical simulations were done for $1310 \mathrm{~nm}$ G.652 fibres of $0,30-0,35 \mathrm{~dB} / \mathrm{km}$ attenuation, effective core area 80-120 um ${ }^{2}$, over 20 DWDM channels with $250 \mathrm{GHz}$ channel separation, $0,085 \mathrm{ps} / \mathrm{nm}^{2} \mathrm{~km}$ dispersion slope. FWM limitations were researched. These limitations were avoided by introducing channel spacing $1,4 \mathrm{~nm}$.

Two channel MIMO-optical camera transmission system was tested on a smartphone. Multiple antennas in wireless communications are used at the transmitter and at the receiver. Serial to parallel and parallel to serial conversions are performed at the transmitter and the receiver. LED is used as data source and smartphone camera is used as a receiver. The mobile device is enabled to receive visible light communications. Several streams of bits can be transmitted simultaneously. The parallel data are transmitted as successive images and decoded to serial data stream. 
Precision time, frequency and phase, bidirectional fibre optic large area, trunk distribution systems are researched at AGH. Master clocks are caesium fountain, hydrogen maser or optical atomic lock. The aim is to obtain remote copies of the atomic clock. Optimization of the link performance, by providing the highest SNR, requires considering nonlinear effects, including Brillouin scattering. Brillouin stimulated optical backscattering originates from acoustic microwave excitation around $10 \mathrm{GHz}$ by high power optical pump. Brillouin backscattering is shifted down in frequency by the acoustic wave frequency. Modelling includes all system components laser source, EDFA amplifier, splitter for power meter, circulator for OSA and multi kilometre non-zero dispersion shifted single mode optical fibre.

Congested computer networks, either optical or any other sending packets, are subject to research at ZUT. Control quality indicators are introduced to monitor the available bandwidth dependency on the intentionally introduced disturbance level. Congestion in communications networks stem from limited bandwidth and hardware resources. Optimal control strategy for maximum utilization of all available resources is the key component in congestion avoidance algorithm. Packets are sent between network nodes via a certain number of intermediate nodes to the destination, including a congested node. If they cannot be forwarded due to limited network resources they are accumulated in egress buffer. There are two contradicting processes. The number of accumulated packets should be sufficient to fulfil the network bandwidth which varies with time unpredictably. On the other hand lowering this reserved value frees more bandwidth. Buffer occupancy can be close to the limiting value or can be underutilized or empty. In both cases the effective network bandwidth is lowered. Correlation between reference queue length and available bandwidth trajectory is assumed. Non-stationary, discrete, dynamical model of data exchange was used for network behaviour simulation and optimization.

Adaptive feedback communication system AFCS is researched at ISE WUT. Growing structural complexity of wireless networks and arrival of $5 \mathrm{G}$ are putting qualitatively new requirements on the physical layer of communication network channels, like: maximal range, reliability and quality, minimum energy consumption, maximal lifetime of energy sources, maximum spectrum-energy efficiency, optimal usage of allocated channel bandwidth, minimal inter-channel interference, elimination of unauthorized access, security, minimal complexity, minimal production and deployment costs. Possibilities to solve these issues have to be addressed by new solutions. Currently the feedback is used only for communications with the end-node user or for transmission of control signals. There is also the possibility of using the AFCS architecture without coding, which can be done by adaptive PA adjusted by the controls computed in base station and delivered to the forward transmitter over feedback channels.

\section{OPTOELECTRONIC AND ELECTRONIC SYSTEMS AND APPLICATIONS}

Maksymilian Pluta Institute of Applied Optics specializes in design and manufacturing of complex optical and photonic measurement systems, as well as solving complex technical issues with building optical apparatus. One of recently developed systems is multi-wavelength laser scattering tomography. Imaging spectrometer observes laser beam waist in the scanned sample. CCD/CMOS camera registers the image. Sample scanning is done for different wavelengths. The samples include among others YAG crystals, GaAs wafers done in different technological processes, glass-spinel boundaries, etc. Observations are done in the same wavelength as illumination or different. The Spectral LST method is a very useful technique for opaque material inspection. Using the diode pumped $\mathrm{Nd}$ :YAG laser and the infrared CCD camera the device is compact and stable. The set of translation stages $(\mathrm{X}, \mathrm{Y}, \mathrm{Z})$ provides the ability to test samples in three dimensions. The system is fully automated and controlled by specialized software. The device was tested using semiconductor materials like GaAs and $\mathrm{Si}$ wafers and ceramic samples. The recorded images play an important role in the material structure understanding and help in improving technological processes of new materials.

There is a need of power quality monitoring in energy systems. Increasing number of power analysers are in use. Institute of Communications offers power quality analyser calibration service, as it possesses accredited metrological laboratory working in electrical, electronic and optoelectronic fields. The lab confirms basic standards in energy quality like supply voltage parameters of energy systems, electromagnetic compatibility, defined for harmonics. The targets of investigation are: power energy quality calibrator metrology verification, traceability of energy quality measurements, and quality of calibration service. Accredited measurement procedures are used.

Jagiellonian PET was equipped with FPGA based real time tomographic data analysis system. High quality image reconstruction generated in real time is of critical concern in dynamic PET. It involves reduction of scan time, gives instant feedback to the operator, focuses on particular body regions, adjusts measurement parameters, enables self-calibration, and instantly identifies any device malfunction. JPET was constructed using the experience and technology from large scale physics experiments. JPET contains nearly 200 detector strips, 400 photomultipliers, over 1500 analogue channels, 50 $\mathrm{kHz}$ readout rate and nearly $1 \mathrm{GBps}$ under full load. The readout system is based on standard TRBv3 master board and 8 slave boards. The MB controls the readout procedure, is a gateway for control and monitoring and synchronies the slaves. SBs do signal discrimination with around 200 TDC each, and direct data output to GbE network. The system constantly digitizes analogue signals and realizes continuous readout. The solution assures no data loss due to preliminary event selection, and reduces measurement time. The costs are high data rates, significant amount of storage, reaching up to TB per measurements and a lot of background noise registered. The required steps towards image reconstruction require data processing and are: data units reassembly into time slots, hit times extraction from binary data, detector geometry mapping, application of calibration parameters, coincidence search in a single timeslot, filtration and LOR histogramming. Next steps are online software data analysis, and full processing on hardware. The readout system based on FPGA chips enables: preliminary data selection and filtration, application of calibration on-the-fly, and true real-time image reconstruction.

New solutions of luminescent lamps were proposed with usage of nanostructural carbon and field emission cathodes 
(IMiO WUT and ITR). The fabricated lamps may be qualified to A++ energetic class. The EEI coefficient of the lamps was 0,04 . The lamps showed initial decrease of luminance due to current instability. Methods of carbon layer deposition and nanotubes growth is researched to improve lamps luminance stability. Motivation of the research stems from energy savings considerations. New energy effective light sources for lighting purposes are still necessary. Field emission is a method of energetically effective electron generation. The applied technology uses nickel-carbon film containing carbon nanotubes in the top layer of the cathodes for electron emission enhancement.

Optical coherence tomography OCT detection systems are researched at GUT. Spectral domain Oct uses broadband light source which irradiates sample and optical analysis using interferometry and spectrometry. OCT advantages include: contact-free, non-invasive, high resolution cross section images, 3D models, high speed and functional extensions. OCTs are time domain, frequency domain, with swept source and spectral domain. Czerny-Turner spectrometer is used in the latter type of OCT with line scan camera as the detector. Functional parameters are: wavelength range, resolution of sensor, speed of data acquisition, length of sensor and corrections of distortions like astigmatism.

Somatosensory feedback technology is researched at Mechatronics WUT for building advanced hand prostheses. Somatosensory feedback, with vibro-tactile stimulator, provides the user of prosthesis with information about the force generated by the used device. The grip force is measured at the fingertips of prosthesis and stimulate user with vibrations. Measurements and stimulations are multichannel. Achieved preliminary parameters were: force range was $0-35 \mathrm{~N}$, frequency range 120 - $305 \mathrm{~Hz}$. The device is subject to further development, especially minimization of the size.

Electromyography EMG amplifier with wireless data transmission was constructed at Mechatronics WUT. The device consists of analogue processing unit of bioelectrical signal, microcontroller unit and PC application. The application has the basic functionalities serving the device. The device is autonomous, battery powered, and registers electromyography signals.

Adaptive sub-ranging ADCs with auto-calibration of offsets of internal components are researched at ISE WUT. There are increasing requirements on precision of adaptive sub-ranging ADCs included inside a VLSI. The question to be answered is how to correct offsets in these ADCs. A method was developed of offset correction using specific features of adaptive subranging ADCs. These features include: output codes are computed in the dedicated digital part using iterative adaptive algorithm, and the amplifier gain may have arbitrary value differing from integer power of 2. Implementation of autocalibration capability requires only small change in the circuit architecture, which is introduction of a switch. The calibration procedure is fully autonomous. The result is reduction of requirements on precision during the VLSI circuit manufacturing process.

Functional driver modules for quantum computer experiments are researched in ISE WUT. Smart electronics is surrounding the quantum core effect to control qubits superposition and entanglement and to manage decoherence. ARTIQ software hardware environment is used and tested which creates advanced real-time infrastructure for quantum physics. It is a high-level programming language describing complex experiments. Enables time critical code running on FPGA and other code running on PC controlling the experiment. SINARA, which is modular, flexible, well tested and open source, is used as high quality hardware for ARTIQ. System architecture includes Metlino which distributes DRTIO commands to other cards in the crate over the MicroTCA backplane in star topology, and distributes DRTIO commands to other crates, receives commands from another Metlino board or control PC. Sayma includes extension cards that contain a FPGA, typically used to generate and process RF signals, ADCs and DACs are on $\mu$ RTM extension, and uses optional FMC extension. Kasli controls external equipment that does not require precision provided by Sayma. It receives DRTIO commands from Metlino master card and up to 12 extensions, which may be connected by ribbon cables.

Data concentrator module is under development for micro satellite family at ISE WUT. The satellites are $10-100 \mathrm{~kg}$ class and for LEO operation. The system applications are: broadband digital communications, direct broadcast services, mobile satellite services, environmental monitoring, and Earth observation. Benefits of using standard mTCA solutions are: short development time, design flexibility, high reliability, full redundancy and low cost. The system under consideration is assembled with: two mTCA carrier hubs $\mathrm{MCH}$ with one redundant, two power units with one redundant, data concentrator unit and mission specific units. The main features of satellite equipment are: possessing backplane with connections redundancy and only conductive cooling with no dedicated electronic units. The main functions are: telemetry storage, downlink transmission data buffering, handling the tele-command data dedicated for further execution and various data buffering tasks. Hardware components are: SSD memory, ZYNQ 7000 series SoC with two ARM Cortex processors and other relevant components like communication peripherals, memory interface controller boot ROM and on-chip RAM. The system is predicted to be micro-satellite on-board class.

Indoor localization system using SDR was constructed in ISE WUT. Existing solutions are expensive and require specialized hardware. Localization may base on several methods like: time difference of arrival or time resolved, dead reckoning, RSSI based, using RFID markers, and UWB based solutions. The assumption is to use cheap hardware readily available on the market. Advantages of using SDR is that all system development is done in software, and it is possible to experiment with various modulations methods and signal formats and frequencies. Time resolved system requires three or more receivers. Time difference of arrival or TDOA hyperbolas are intersecting each other, and the traced object source is located along multiple hyperbolas just at their intersection. TDOA has to be estimated. Additional transmitter with precisely known distance to each of the receivers may be added to achieve time synchronization of the whole network of base stations and the localized source. Conventional approach assumes maximization of the value of cross correlation. Matched filtering assures better accuracy, there is no need to transmit signals between base stations - which is a serious advantage in real-time solutions. However, a model of the received signal is needed, which makes the method quite difficult because of calculation load. TDOA estimation accuracy depends on the modulation and SNR. There 
is no analytical solution for more than four receivers, but the more receivers the better accuracy of localization. Closed form iterative multilateration and optimization method is used. These methods do not guarantee convergence, but can be used with any number of receivers, they easily extend to Kalman filter and are easier for implementation. Formal description is by overdetermined set of equations and is a nonlinear least-square problem, which may be solved by Gauss-Newton method. The minimized function is non-convex and can have multiple local non-local trapping minima. For poor TDOA estimates the error converges to some value but most likely not at the global minimum. Hardware was set up with evaluation board STM32F4 microcontroller, SX1272 modular transceiver, transmitter frequency range $860-1020 \mathrm{MHz}$, modulations (G)FSK, (G)MSK, LoRa, and OOK. System management and receiver base station hardware by a single board computer Raspberry Pi and real time operating system ChbiOS, with rtlsdr module $24 \mathrm{MHz}-1,7 \mathrm{GHz}$, and 3,2 MSPS.

A satellite onboard computer $\mathrm{OBC}$ is under design and tests for LEO missions applications. OBC responsibilities are: control all the onboard systems of the satellite, receive and interpret commands from ground stations including protocol security aspects, automatic tasks based on built-in schedule, meeting many requirements in terms of radiation tolerance, reliability, availability and safety. It is a part of short time to the market HyperSat project, which is a modular, versatile satellite platform based on commercial, cheap COTS components. Hardware platform is realized on ZedBoard Zynq 7000 ARM/FPGA SoC development board with Linux/ Android/ RTOS development.

\section{AUdio, VIDEO AND VIRTUAL REALITY TECHNOLOGIES}

Real time pitch shifting for audio signal, which was guitar sound, was designed using a general purpose microcontroller ARM Cortex (ISE WUT). The following sound features were considered for audio signal processing: loudness via signal amplitude, pitch - via the fundamental frequency, timbre - via harmonic structure, time envelope. Pitch shifting included changing the pitch and preserving the original time duration. Audio signal was modelled as sum of sinusoids with amplitudes varying in time, and pitch shifting by multiplying frequencies. Phase Vocoder algorithm included signal analysis and estimation of spectrum, modification of phase spectrum and synthesis via inversion of DFT blocks, employing OLA process with altered overlap factor and resampling.

A team from IRTM WUT carries research on image processing. Colour transfer is used in image processing by fitting clouds of colour points. Generally, colour transfer is image processing method that can alter colour appearance in the input image by borrowing colour statistics from the reference image. Colour transfer may be numerically treated as mapping function of the set of colours from reference to input image. The technique is used for colour correction, greyscale colourization, and style transferring.

Cheap virtual reality headset with extended content for mobile devices is researched, where mobile sensors are used to rotate camera in the VR scene. Extended video content is detected in region of interests. Object disappears when region of interests and timestamp are different. VR player connects to web server, which holds videos, thumbnails and sequence description. Video events are emitted to player with additional content from dynamic asynchronous resources.

Face recognition systems contain smile detector correlation ability. Application bases on SVM classifier, 2D and 3D mouth area classifiers for different image frames. Detection is done for several tens of facial salient points for non-smile images. Mean image is calculated of training images for non-smile class. Detection of several tens of facial salient points is done for smile images. There is a possibility to train a simpler or lighter detector. OpenCV and dLib algorithms are compared for SVM based primary smile detector. Mouth indexing uses full collection of salient points in addition to cheek, nose, chin and both eyes indexing. SVM performs two-class classification smile vs non-smile. Mouth area is calculated via 2D triangulation, similarly to face oval. Action units are applied for smiling 3D model. Action units include lip raisers and stretchers, etc.

WebGL and WebAudio software in lightweight edition were used for multimedia education (IRTM WUT), including graphic effects, image processing, shaders, audio processing, etc. Light weighted servers like CherryPy and Node.js and web wiki editor were used, which creates a minimalist Python web framework. The environment automatically integrates of code fragments. Also work is done on automatic classification of input images.

Heavy motorway traffic requires a lot of data acquisition related to safety, management, optimization, cost rationalization, etc. Remote location sensing optoelectronic system was implemented for vehicles counting and classification. The system aimed at cost efficiency, cheap solution and based on video stream analysis. The solution is based on: implementation of algorithms for automatic digital image analysis within network of hybrid systems of video cameras, applicability for various weather conditions, and practical test implementation. Data acquisition is done locally and data processing is centralized. In final solution there are predicted two image data sources optical and microwave. System requirements are as follows: compactness, weather resistant, ability to work off line, local data storage for at least two weeks, data acquisition distance tens of $\mathrm{m}$, bitrate around 1 Mbps. The system opens ability of testing of various algorithmic approaches combined with vehicle detection, tracking, and classification and dataset calibration.

Cave type automatic virtual reality systems are researched at GUT in immersive 3D 6W visualization laboratory. Full space geometry, illuminating and tested sections, and directional characteristics are used for modelling including space vectors and all angles horizontal and vertical. Two types of relative directional luminance distribution were tested wider and narrower. Performed simulations show that distribution of luminance is strongly dependent on the directional characteristics of the screen material. Tracking system was designed for the cave with glasses equipped in passive markers. Accuracy of the tracking system was evaluated.

Data and physical phenomena visualization is done for the ALICE experiment at II WUT. 3D effect can be obtained in several ways by using virtual reality equipment like Oculus Rift, 3D monitors with active or passive glasses. Monitor based approach lacks interaction with the viewer, because content do not move with moving viewer and no real window behaviour is observed. Using multiple displays, the content should move from display do display. Other characteristics possess VR 
headsets which offer noticeably better impression of immersion and are optimized for games. VR headsets badly visualise wire based scenes because tracks are basically lines. Several techniques were included to better the visualization like texture size adjusting and smoothing, vertex shader approach, etc.

Image tracking software for applications in dynamic athletic sports is under development at ISE WUT. An example is localization of wushu players. Machine learning systems enables the designer to create own performance and predict athlete location. Proposed method of athlete localization include: creating platform pattern, comparing frame with platform, locating the athlete and filtering results. There are several methods to create platform pattern: find a frame with empty platform, create numerous snapshots of performance and simulate long exposure or apply median filtering. There are several methods of comparing frame with pattern: histogram and image difference. The athlete if located by finding the biggest blob inside the platform area in a reasonable distance from previous one. Results filtering includes: application of low pass filter to restricted area of results, or application of Kalman filter. Localization improvement involves learning image momentum of the athlete and compare with blobs, and compare single object tracking algorithms introduced in new OpenCV library.

Multi-standard video signals integrator VSI is under implementation for security and safety purposes, and coordinated by ISE WUT. Goal of the project is: integration of video signals from different video management systems, providing access to video streams via a central server with appropriate access right control, and provide the authorized users with possibility to control PTZ cameras. VSI architecture includes main components which are: console, video streaming servers, PTZ servers, video sources connected by NVR and PTZ adapters including industrial VMS and VSI capture device. VSI console has three layers: administrative, operators and passive. Administrator manages other consoles, assigns the access rights and manages video streams. Operator console lists available streams, displays up to 16 streams, configures display screen, localizes stream source on the map, controls PTZ cameras, has joystick support and screen switching ability, and creates directory of signals. NVR server is implemented in $\mathrm{C}++$ and has all relevant versatile functionalities and flexible configurability. RTSP server optimizes bandwidth usage. Bandwidth is adopted if the source is capable of providing several streams with various bit rates. PTZ server provides unified access to different PTZ protocols used by cameras. ONVIF and VISCA adapters are currently available. VSI capture device platform is developed using ZCU board with FMC extension cards. The used technologies are: FPGA ultra-scale, embedded ARM in FPGA, H.264/AVC video compression, multi-interfaces - HDMI, BT1120, AXI, Ethernet and $\mathrm{I}^{2} \mathrm{C}$, and fast external memories DDR4. Software environments are Petalinux and Vivado for Xilinx. The prototype implementation of VSI was created with functional and scalable laboratory demonstrator. The system is ported to final hardware format.

\section{ENERGY MANAGEMENT}

Energy consumption by a large number of individual smart appliances may be optimized using micro smart grid technologies. The aim of power oriented smart grid is to increase the efficiency, reliability, economics, and sustainability of the energy production and distribution. The general energy grid has hierarchical structure and contains the following levels: central and local, where local systems are split to numerable home area networks. The latter are developing tremendously containing dozens of various networked devices called smart appliances and this number abruptly grows. Modelling of smart grids includes assumptions of predicted min and max number of appliances, min and max power they consume, hours of operation, customs of users, time of year, weather conditions, and so on. Algorithms may be applied to reduce energy consumption by the local grid at home as well as city quarter levels. Management of a flexible local grid includes also local energy generators (wind turbines, photovoltaic panels, hydropower, if any) and storages (large batteries) and their characteristics like power and time availability - if they are temporal. Usually the aim, in the simplest, first order time domain optimization approach, is to reduce the peak demand, appearing during the 24 hour consumption cycle. Analytical model of the grid includes all reasons of energy consumption reduction and vice versa the consumption increase. The family of quality indicators include the ratio between momentary demand to the power supply. Some confinements are imposed on the changeability of this value and acceptable difference between the power demand and supply, to keep the grid stability. Smart grid uses long term data on power usage to predict the nearest future. Cloud services are employed to control home appliances over the Internet, which is include in the grid model. Using the model, the energy usage may be, to some extent, equalized during the night and day cycle, by spreading the energy usage peaks to the neighbouring regions. The energy usage equalization work is done at Uni. ZG.

Energy usage equalization system by load shifting algorithm was implemented in FPGA/vhdl hardware. Load shifting algorithm for the domestic daily load curve relies on classification of loads and analysing the possibilities to flatten the evening peak to the afternoon and night valleys. Thermal power accounts for about $85 \%$ of total installed power facilities in Poland. Critical situation is observed during summer when these very large facilities lack proper cooling and generate less power. Power generation in winter is thus $30 \%$ bigger. There were identified all factors which potentially increase and decrease power demand, and conditions of their work, especially distribution of power consumption in time. The evening power consumption peak (18-21 h) is mainly associated with the communal and municipal users. This peak can be potentially reduced locally by tariff schemes via price zones, and stimulus programs via reduction on request, etc.

Energy harvesting EH is a very current research issue, under consideration in numerable aspects. Energy harvesting systems driven by human walk include: piezoelectric boots, vibrating batteries, piezoelectric straps, vibrating generators, biomechanical devices for example associated with knee and other joints, smart fabric, self-winding watches, energy harvesting backpack with flexible solar cells and inertial devices with springs and mechanical rotary and/or linear electrical generators, and many other solutions. The idea of energy harvesting by human walk is to get frequently small amount of energy from mechanical source. Small autonomous energy harvesting system associated with a human requires sensors, energy harvesters, communication, energy transport, portable 
computing and management devices, energy storage, etc. Wearable EH device is usually associated with other parts of the on-body functional system like: monitoring health state of climbers, soldiers, disabled persons, humanitarian workers, also powering, etc. EH device supplements such functions like charging biomedical sensors, charging energy storage, power banks, batteries for night vision equipment, thermo-vision, radar, radio and wireless data communication devices. Combination of several energy sources co-working with EH can provide today autonomously up to several tens of $\mathrm{W}$.

Research on using solar energy for cooling purposes is carried at LUT. Solar energy availability is in quite good synchronism with cooling demands. Typical examples are thermoelectric TE Peltier modules powered by DC directly from photovoltaic panels. Such technique is used in wide cooling applications ranging from electronics, medicine, military, industry, in particular batteries and cabins in electric cars. The main advantages of TE cooling, which promises for their extended applications are, especially in higher power cooling demand situations: compact size, lack of moving parts, low failure rates, no noise and vibration, precise temperature control, no harmful refrigerants and chemistry, operable by DC - thus may be directly powered by the PVC. An experimental system was built to cool a small room. The research concentrated on energy consumption, cooling efficiency and system optimization. System efficiency is determined by the cooling efficiency of the hot side of PE modules. Hot side may be used for in-house water heating.

LUT continues research on energy cogeneration technologies and distributed energy applications. Energy saving is one of the priorities of energy policy in Europe. There is observed increasing usage of renewable energy sources, what changes considerably the conventional energy industry. Use of the distributed generation systems widens. There are various installations where owners derive benefit only from energy savings, in contrast to energy which owners of installation would have to buy in the conventional energy system. These are installations where the energy demand of the owner is smaller and the owner gets profit from energy reselling to master network. Other configurations are when there is some balance between owner usage and resale, and finally when the owner is larger local producer of energy. The energy needs of microsystem loads may be covered from power networks, energy storage and generators working in polygeneration. Offgrid mode installations using only cogeneration in constant proportions of heat and electrical power may be not sufficient to maintain power stability of micro energy systems.

Electrical appliances are characterized in transient state (IRTM WUT). Energy consumption is measured of individual appliances. Non-intrusive appliance load monitoring system NIALMS is used in smart home. Network node measures instantaneously current and voltage, with the system controller as wireless home PC. Each device is modelled. Each event is recorded with identification algorithm. Appliances are characterized by time signal changes of energy usage. Signal has transient parts and steady state parts. Transient features are observed when devices are turned on with the current waveform depending on voltage phase in power grid. Local grid is characterized by long term recording and analysing the power usage curve.
Availability model of stand-alone off-grid photovoltaic system was researched at ISE WUT. Basic features of off-grid solar system are: simple and low-cost design, low voltages 12$48 \mathrm{~V}$, requires a battery, electricity cannot be sold to grid, and relies only on solar power. Applications of stand-alone PV systems include: traffic signs, road appliances, road radars, intelligent city equipment, water buoys, parking machines, city bike system, and many more. Sizing the PV system is associated with selecting nominal power of PV module required to produce enough electricity to power up the designed loads, and includes estimation of daily power consumption, solar irradiation, and PV efficiency factor and orientation. These estimates define required nominal power of PV module. Average daily solar irradiation, and coefficients of PV module tilt and temperature, assuming constant power efficiency coefficient, can be obtained from databases for particular localization of the PV module. Solar radiation databases collect irradiation values from satellite images and meteorological measurements. Open access web services are available in this domain resulting from international research programs. Some of these databases are: NASA-SSE, PVGIS, HelioClim, and Solargis. Apart of PV sizing the reliability has to be estimated. Experimental solar system was constructed with the aim to evaluate real PV energy production statistics. System assumptions were: low cost PV module, south orientation, roof installation with no shading, continuous measurement of PV energy, long time uninterrupted measurements, taking into account all real factors like temperature, dust, snow coverage, rain, etc. There were calculated daily generated energy and global tilted irradiation GTI. The procedure included measurements and calculations of: daily PV energy produced, solar module nominal power with standard irradiance, estimated daily sum of GTI, estimation of PV energy generated in any solar module installed at the same location. Daily production of energy in a PV system with assumed probability was calculated from daily sum of irradiation. PV power source availability equation was derived for location in Central Europe. It is possible to apply this method to other locations using datasets from satellite solar radiation databases.

\section{HIGH PERFORMANCE COMPUTING AND COMPUTATIONAL INTELLIGENCE}

A novel approach was proposed involving the rule based logical model of the control process for the following system development stages: formal verification using model checking technique, synthesis in FPGA chip, and implementation in AVR microcontrollers using $\mathrm{C}$ language. Logical model ensures consistency between a verifiable model, VHDL and C models. The aim is to fully automate $\mathrm{C}$ code generation. Implementation tests were done with ATMega 162 microcontroller at UZG. C code was generated from Petri based specifications of logic controller. Justification of the research was that event driven systems appear currently in many domains of our life. Controller specifications were defined as a first stage of the design. A control process was specified as an abstract rule-based model describing main components of state changing. Model contains the following sections: variable definition, assuming initial values for variables, transitions descriptions, and finally input and output signal changes. 
A method of micro-operations encoding was proposed for LUT based Mealy finite state machines. The method is based on methods of structural decomposition of FSMs to diminish the number of arguments for input memory functions in the output functions. It is essential to reduce the number of functions depending on $\mathrm{L}+\mathrm{R}$ arguments by transformation of state codes, and reducing the number of arguments in output functions of FSMs by encoding the fields of compatible micro-operations. The method is an alternative to encoding collections of the output functions. For numerable conditions a single LUT is only necessary to generate any function with required number of arguments. In other case there are needed more than a single level of logic in the circuit to generate the output functions. There is a statistical relation between these two cases. Amount of needed hardware, propagation time and power consumption should be added to the model to implement final FSM model for given GSA in the FPGA chip.

Recently reversible circuits have attracted attention as components of post-CMOS technological background for quantum algorithms, also in photonic and nano-computing passive, no-gain technologies. Reversible computing is a model of computing where the computational process to some extent is reversible, or time invertible (II WUT). A necessary condition for reversibility is that the relation of the mapping from states to their successors must be one-to-one. To implement reversible computation, estimate its cost, reversible gates and methods of circuit synthesis are needed. An inverter gate is reversible because it can be undone. The Exclusive Or, XOR or AND, gate is irreversible because its two inputs cannot be unambiguously reconstructed from its single output. A reversible version of XOR gate (CNOT, controlled NOT) is made reversible by preserving one of its inputs. Reversible gates have no more inputs than outputs, thus there are no fan outs and loops. In the Turing machine model of computation, a reversible Turing machine is one where transition function is invertible. Each machine state has at most one predecessor. This is of course a considerable simplification of the general situation in digital logic. Usually the reversible circuits are synthesized from NYT gates library (NOT, CNOT and three variant CNOT Toffoliwhich preserves two inputs). Reversible circuits are described by balanced Boolean functions, which have 0 minterms equal to 1 minterms. Reversible circuits are constructed of three-input and three- output Fredkin gates. Any logical or arithmetic operation can be constructed of FG. The research concentrates on usage of standard C-language string functions for reversible circuit and sub-circuit design and manipulation.

Reversible circuits can be implemented in FPGA chips. Implementation of projects containing reversible components requires expensive components realized in quantum technology of particular choice. Quantum technologies are said to be ideal technologies for building cipher and decipher machines. Such machines require usage of reconfigurable reversible gates and circuits. The fastest way to verify the model based on reversible gates is to implement it in the FPGA structure, and such research on various design methods is realized in II WUT. The feature of reconfigurability stems from the ability of the gate type determination by additional control lines. Test structure was implemented in Altera Cyclone V chip. Developed test bench confirmed the correctness of the design which may be applied for further development of quantum systems. Reversible circuits were also synthesized using S-maps of identity functions.
The problem of synthesizing incompletely specified reversible functions is of importance in many areas like design of: incomplete oracles for machine learning applications, functional blocks included in larger oracles or spectral transforms where "don't cares" occur similarly to classical network design, reversible state machines and quantum automata. A question is asked how to determine the "don't care" assignment leading to the circuit with minimal cost. To answer the multilevel logic synthesis was applied. Decomposition to reversible synthesis includes: decomposition of large nonreversible function to small non-reversible ones, embed individual small functions i.e. turn them into small reversible ones, optimally implement small reversible functions, recompose reversible functions in the same way the nonreversible ones were composed. Functional decomposition splits a Boolean function into a network of smaller functions which are easier to analyse and synthesize. Exact reversible synthesis can be done optimally to as small and as simple functions as necessary. "Don't care" assignment done during decomposition influences positively the quality of resultant reversible circuit.

Minimization of cost measures of synthesized circuits is a research issue. The following criteria are conflicting: gate count, quantum cost and number of lines. A trade-off between these criteria is required. The major focus so far is put on synthesis with optimizing quantum cost, where some increase in gate count and number of lines is accepted. Algorithms are developed at II WUT to assume some standards to minimally increase these numbers. The algorithm includes: conversion of reversible function to PPRM expressions, grouping PPRM terms for finding common terms among component functions, realization of PPRM terms with MCT gates, inserting CNOT gates for transmitting signals between circuit lines, and finally reduction of quantum costs of the sub-circuits. The algorithm was proven to generate circuits with smaller quantum costs.

True random number generators are necessary for safe communications, cryptography, SCA countermeasure, math statistics like Monte-Carlo methods, and many more. TRNG is used in public-private key communications. Pseudo random number generators are not enough because they use deterministic algorithms instead of stochastic processes. Algorithm type determines the generated number. TRNG is generated as thermal noise of a resistor, jitter of a generator like ring oscillator, PLL noise in FPGA circuits, bistable instability in vicinity of metastable solution, etc. The analogue noise has to be sampled and converted to bit-stream. The advantage of metastable TRNG like single bistable flip-flop is that the output signal is digital not analogue. The generated bit stream is random. The disadvantage is that metastability is sensitive to fluctuations of the initial conditions. The work on TRNG and FPGAs is carried in ISE WUT. Presence of radiation is researched on the quality of TRNG randomness. Metastable based solutions are vulnerable to EM field attacks. A hybrid solution is proposed containing ring oscillator and metastable flip-flop. The solution was evaluated using NIST standards and was shown to fulfil them properly.

\section{GENETICS, BIOMEDICAL APPLICATIONS}

One of the genetics specialization of Department of Plant Genetics of SGGW is research on cucumber. Assembly of 
cucumber somaclones are performed. These are variations seen in plants that have been produced by plant tissue culture. Typical genetic alterations are: changes in chromosome numbers, chromosome structure and DNA sequence. A typical epigenetics related event would be gene methylation, which is a method for obtaining new traits in agriculture and horticulture. The method includes; next generation sequencing, DNA isolation, De novo assembly and genome completeness analysis by BUSCO. Assembly includes trimmomatic of contigs and scaffolds and BFC.

New tool was designed at II WUT to assemble repetitive regions using next-generation sequencing data. DNA assembler uses graph theory algorithm to convert set of reads to set of contigs. The most popular methods are: overlap layout consensus OLC, greedy algorithm and de Bruin graph DBG. Many contigs but not one are created by assembler because there are read sequencing errors, and repetitive sequences are longer then read by OLC or than graph dimension by DBG. Additional assumption is that the reads are distributed uniformly on the source sequence. DNA assembler vertex is unambiguous, no new conting, when it has only one input and/or one output edge, and in other cases is ambiguous, i.e. creates new contig. Vertex is treated as unambiguous if it has zero, one or two inputs (output) edges and, in the case exactly two input (output) edges exist, for one of them simple return path exists. The implementation was done in client-server model with the thin client architecture. Three later software was designed including presentation, data processing and data storage, plus GUI. Source code as well as demo application with web interface and additional data is available online. Application was used to assemble human mitochondrial DNA, amplicons of human genome and some other animals and plants.

Optimization of artificial gene is researched at II WUT. The purpose is: immune protection, enzymatic catalysis, transport and storage and motion. Available methods of artificial gene synthesis are: chemical, polymerase chain reaction PCR, and ligase chain reaction LCR. Evolutionary algorithm has all necessary features like: individual synthesis protocol, initial population, mutation, proportional selection, fitness function, termination condition by declaring number of completed iterations. The advantages are: shortening of calculation time, use of GPU to predict secondary structure, dynamic calculation of fitness function parameters, include short chains identity to optimize simulation, freely available in DNA synthesis web, and availability of demo server.

Dedicated software is developed to detect genomic rearrangements using markers in II WUT. Rearrangement detection phases include: defining markers, searching for markers, and finding rearrangements based on markers positions. Approximate pattern search includes; calculation of cross-correlation, pattern position are found where crosscorrelation is above threshold, and discrete Fourier function is computed with FFTW library. Algorithm correctness is verified by detection of single and multiple rearrangements. Execution time depends on sequence length and number of markers. The research goes into the following directions: new marker definition algorithm, more efficient markers search algorithm and new rearrangement detection algorithm.

New DNA assembler Katome was implemented in Rust at II WUT. Search for new assemblers stems from problematic concurrency of existing algorithms caused by hidden mutable states and data races, as well as high memory usage. Rust programming language guarantees memory safety, supports for numerous platforms, provides minimum runtime and is strongly typed. Katome implementation features are: modularity, based on the interface composition including traits, is extensible by design, has optimal algorithms for graph building and collapsing, and has simple and clear configuration based on text file. Implemented Katome was compared with dnaasm.

Laser capture microdissection LCM was used to study flower morphogenesis at SGGW. This is a method to rapid procure subpopulations of tissue cells or even single cell under direct microscopic visualization from heterogeneous tissue. Capture cap with thermolabile plastic film is covering the culture. Laser pulse catapults cells of interest. Cells of interest are on thermoplastic film. LCM does not change or damage the morphology and chemistry of the sample collected, neither the surrounding cells. It is a useful method of collecting selected cells for DNA, RNA and/or protein analyses. It can be performed on a variety of tissue samples, even on living cells or organisms, cell smears, chromosome preparations and plant tissue. The method is tested for prediction of genes involved in the development or inhibition of the growth of specific flower organs, and at which stage of bud growth it occurs.

Bioinformatics and expression analysis of DNA is used for creation of new variety for horticulture, agriculture, and forestry. More efficient food and seed production are searched. Obtaining new varieties of features is looked for in the production of biomass and biofuels industry. Practical purpose of the research at SGGW is to point, isolate and characterize cDNA sequences of genes which take part in flower morphogenesis and flower sex differentiation. Differentiation is combined with collection of such sequences which are present only in one of the analysed pool of transcriptomes. High selectivity of biomaterial choice indicates that obtained sequences are differently expressed between flowers of different sex. Bioinformatic tools provide opportunities for development good characteristics for new genetic and genomic resources.

Genomecmp software enables detection of genomic rearrangements in plants. The aim of research at SGGW is to check and investigate rearrangements/variants of DNA sequences including type of rearrangements, position across the DNA sequences, frequency of occurrence, characteristic feature of rearrangements. The research aim s are: genome structure, comparative genomics, how genomes are related to each other, evolution - how genome change and create new species, which rearrangement is correlated with specific features, why organisms differ to each other. Genomecmp provide several types of presentation of results: summary, detailed tabular data and graphical, and by this enables better insight in dynamics present in genomes and mechanisms responsible for evolution.

$\mathrm{CuGene}$ was used as a tool to view and explore genomic data at SGGW. CuGENE functionality is as follows; display of sequence and annotation tracks, interactive zooming, editing of individual features, supporting manual annotation, intuitive aligned sequences easy to view, many searching algorithms, cooperation with other applications. It is configured as clientserver application, and enables storing of many organisms, and many genomes. It was extensively used for cucumber genome, and the results are freely available on the cugene web.

Investigation of different types of laser irradiation with biological tissue phantoms is done at GUT photonics lab. Work 
aims are: better judgement of influence on particular parameter of phantom in different parts of radiation spectrum, and possibility of producing more precise optical tissue phantoms for specific laser. Phantoms are done with PVC $\mathrm{ZnO}$ and different inks. Several types of lasers were used for phantom tissue irradiation like: KTP and Nd:YAG,

Mobile optogenetic module was designed for research application in mice by UW and IBD PAN. Optical part of research is combined with activation spectra of channelrhodopsin ChR2 and halorhodopsin NpHR with good spectral separation which as a result gives possibility of activation of one protein without activation of the other. Fibre optic least invasive catheter with sharpened tip was used to penetrate the brain and deliver locally optical activating power. Also LED attached to the end of cannula was applied. Behavioural experiments were performed with optogenetic modules. Implantable optoelectronic modules with LED for optogenetic stimulation of living mice brain tissue has been developed. The devices have been tested in vivo for ventromedial nucleus (VMN) and arcuate nucleus (Arc) of the mouse hypothalamus by infecting the entire structure with channelrhodopsin and it was observed a striking change in the jumping activity.

Can a mouse distinguish between cardinal and oblique orientation, is a question researched at Nencki Institute. This difference can be registered during in vivo experimental technique, which is intrinsic signal optical imaging ISOI. This technique visualizes functional representation of large cortical region in the brain. During the activation of neurons the level of oxygenated blood increases what can be distinguished optically by $\mathrm{HbO}_{2}$ and $\mathrm{HbR}$ colorimetry. The technique requires only skin removal over visual cortex because the scull is very thin and semi-transparent. Glass window and agarose film is a necessary and sufficient optical interface. Blood vessels in the visual cortex are clearly visible and exposed. Young anaesthetized mice are subject to experiments. The monitor screes shows black and white gratings of four basic orientations and the CCD camera observes the changes in the visual cortex blood oxygenation areas. Temporal profiles of hemodynamic maps were recorded and analysed. It was shown that the mice brain responds differently to cardinal and oblique orientation simulations.

Research on vision response (by measuring visual evoked potentials VEPs) in rats to visual cortex stimulation by signals of various frequency is done in Nencki Institute. HF simulation is responsible for loss of vision. Laboratory set up consisted of: linear electrodes $25 \mu \mathrm{m}$ tungsten nanowire 12 channels, hardware filter $0,1 \mathrm{~Hz}-500 \mathrm{~Hz}$, sampling rate $1 \mathrm{kHz}$, recording in visual cortex of LED flash stimuli $1 \mathrm{~Hz}$ and $7 \mathrm{~Hz}$.

Electromagnetic positioning system is constructed for freely moving animals in an intelli-cage living experimental environment. The system consists of coil matrix supply by coil drivers and readout by CPU and FPGA. Mouse is implanted with a localization sensor.

Measurement system for contact thermography is under construction in ISE WUT. The main aim of this project is to design and build a practical cheap, easy to use device for contact thermography, which is to be applied for improving allergen testing and for other medical purposes. Allergen in micro-drop application is placed on skin and positive test makes the skin red and itchy and with elevated temperature. The portable device is manufactured on flexible PCB, and uses diodes arranged in matrices for measuring the temperature and for heating. The sensor matrix is connected to miniature controller box which is tied to arm. Initially the diodes parameters are not precisely known including nominal voltages and temperature coefficients. These characteristics have to be measured a priori i.e. temperature characteristics and then nominal voltage and temperature coefficients corrections. Knowing these parameters and having them saved in the microcontroller system it is possible to make precise skin heat map. Measuring process includes the following stages: system calibration, measuring skin temperature map, and sending data to PC for map processing and displaying, and input data to diagnosis supporting system. Measuring process includes: measuring diodes voltages at patients skin temperature, calculating the temperature from equation achieved during calibration, setting this temperature as a reference level, measuring the skin temperature after putting allergen on the skin. Atmega hardware was applied for building the device demonstrator. To calibrate the device a reference thermo isolated device with thermostatic circuit was built.

Time-frequency decomposition and linear discriminant analysis was used for classification of electroencephalographs at ZUT. International system of 10-20 electrodes was used on the scalp for typical non-invasive measurements. The following issues were addressed during the EEG measurements: artefacts, and brain activity deformation by different heterogeneous media distributing the interferences. EEG signals are non-stationary and random in nature, and are considered quasi-stationary in short time periods. Traditional frequency analysis is not appropriate for the measurement and processing of biomedical signals. Short-time Fourier transform for a given signal in relation to the window, was used instead. Linear discriminant analysis provides more class separability and calculates a decision region between given classes, and creates linear combination of data sets which yield the largest mean differences between the desired classes. Two pathology cases were researched epilepsy and Alzheimer disease, and the proposed technique showed good performance in case separation. Linear classification can successfully be used in detection of pathological changes in the EEG signals.

\section{BIG RESEARCH EXPERIMENTS}

Big data is a critical issue in high energy physics experiments. The world of science is struggling with the overflow of data for a long time. After launch in the 2000, the SDSS telescope with total datastream $200 \mathrm{~GB} /$ day, gathered more data in a few weeks than all the previous astronomy detectors combined. This trend is continued and even strengthening in all big research experiments concerning gravitational waves, dark matter and energy, astroparticle physics, biology and medicine, etc. Big experiments, big data, big throughput, these are inherently combined features. CMS experiment has 150 million sensors, transmits 40 Mega-frames per second, observes 600 million collisions per second and sees $1 \mathrm{~Pb} / \mathrm{s}$ of data which is hundred times more than all the Internet nodes combined. To cope with such a mountain of data, extreme and intelligent selectivity has to be implemented in the data selection processes by the DAQ system. Even the selectivity of the order of $10^{-12}$ leaves the experimentalists with $1 \mathrm{~GB} / \mathrm{s}$ of archived data, which is $30 \mathrm{~PB}$ 
per year. Till now the experiment has gathered hundreds of $\mathrm{PB}$ of data to be yet processed off-line for precision results. Collected data keeps growing and in a decade it will hit a mark of EB for a single big experiment. LHC computing grid connects over 40 countries, 170 data centres and 8000 users. LHC@home action was launched, similar to the initial idea of SETI@ home. Distributed computing is used by numerable large science projects to cope with large data sets. Data to be analysed off-line are divided to small autonomous packets and distributed to numerable individual volunteering project participants. The project is open and everyone can participate. All the volunteering participant has to do is to install BOINC application and join the project. Big and the large scale is beautiful. $10^{5}$ or more PCs participating will do the change. The numerable calculation issues to be solved are among others pileup mitigation with machine learning, overlap muon track finder, etc. Big data has been a well-known phenomenon in the world of science since many years. HEP challenges in this domain are essentially different from the typical meaning of Big Data, and especially what is understood by industry and social sciences. LHC is definitely a world class leader in Big Data science and applications. The difference is visible by watching more closely the industry approach which calls for collecting everything, run analyses often via NN, visualize data and profit.

Large research experiments require fast events, data and some slow processes synchronization of enormous quality. Synchronization is ambiguous entity. We look here for clock and phase synchronization via the distributed timing system, synchronous data transmission, data packets synchronization, time synchronization, timestamping of data packets and experiment events, timestamping of apparatus activities, etc. Clock synchronization embraces frequency synchronization or syntonization, and phase synchronization often achieved through clock recovery circuits. Two independent master clock sources of exactly the same frequency require synchronization. Clock distribution network is a major part of synchronization. Precision clock signal, with no ijtter and with phase control, has to be distributed to the whole large experiment to all distant and relevant locations. Master clock is isolated from the endpoint by a single or concatenated clock buffers. Experiment data transmission system is multi-channel and packeted. The packets are aligned in time via synchronization markers located in headers, as they have variable length and originate from different sources. In numerable cases synchronous serial data are sent through high speed asynchronous links. Asynchronous clock has to be synchronized with synchronous clock via defined relation. Disadvantages of this type of transmission is that additional synchronization is needed and there is higher risk of synchronization loss. In the source synchronous links clock signal is transmitted with data. Essentially there are two choices, either the clock is sent via a separate line or is embedded in data stream. Now the prevailing trend in HEP experiments is to embed clock data recovery directly in the unified transmission system. However, most of practically exploited systems use mixed synchronization technique. Higher level layers are usually either synchronous or asynchronous. Front-end components are mostly source-synchronous. A general trend is towards source-synchronous systems like GBT and White Rabbit. Master clock in mixed-mode systems communicates directly with FPGA and further via CDR to ASIC.
Data acquisition and synchronization system is under construction for the CBM experiment at FAIR. Impact of heavy nuclei with energies up to several tens of $\mathrm{GeV}$ to a target recreates conditions similar to these in the interior of neutron stars. CBM experiment characterises by continuous work at high frequency up to $100 \mathrm{MHz}$, presence of multiple detectors, lack of external triggers, and event identification based on aggregated data. The functions of constructed system are: collecting data from multiple low speed links from detection circuits, ordering and packaging data into micro-containers covering specified time intervals, sending packed data through high speed links, distribution of synchronization signals, control commands and configuration. The latter two classes of signals have to be sent with deterministic latency. The system is realized on AMC FMC Carrier Kintex, AFCK board, using open hardware standard MTCA.4 architecture using collaboration agreed firmware. White Rabbit is considered as a reference clock source for the DAQS. Clock quality will be demanding thus jitter cleaning will be necessary via phase detector and loop controller.

Hardware platform for CBM common readout interface is under design in ISE WUT in collaboration with GSI. The new design originates from the initial concept of the CBM readout. The experiment is serviced in the CBM building from both sides CBM detector cave $100 \mathrm{~m}$ distant, and computer centre $700 \mathrm{~m}$ distant, over optical links. The DPB firmware blocks were tested in the STS-XYTER tested which uses the black-box model of the GBTx and E-links and IPbus-based readout with limited bandwidth. The tester is under integration with GBT. To test the operation of the GBTX ASIC in modes needed in the STS/MUCH readout 160/320 Mb/s, a dedicated AFCK-GBTtester has been built. The requirements to the CRI board are as follows: handling required number of GBT links to receive data from the CROBs, providing sufficient PCIe bandwidth to send received data to FLES input node, provide sufficient amount of logical resources to concentrate data. Input data bandwidth analysis was done and relevant optical transceivers indicated, FPGA chips chosen, together with cost analysis.

FPGA chips are ideal candidates for interfacing the FEE in the CBM DAQ system. They offer flexible communication interfaces either supported with dedicated cores or possible to implement in the programmable logic. There is a possibility to operate the system in hard real-time, thus fully deterministic precise timing is achieved. The disadvantages are: high cost of FPGA solution, difficult implementation of more complex data processing algorithms and communication protocols, especially related with huge amount of data buffering and repeated retransmission. The optimal solution is to use FPGA plus $\mathrm{PC} / \mathrm{CPU}$ in DAQ. Efficient data delivery from FPGa to PC memory is via usage of DMA. The FPGA implementation offers good opportunity to prepare DMA system adjusted to the specific requirements of the particular DAQ. The solution is based on Xilinx provided IP cores. Similar blocks are available also for FPGA or SoC chips from other vendors. The used Linux kernel is also useful and portable to other hardware platforms.

One of hot plasma research directions is absolute measurements of impurities which subtract plasma energy. Such a system is constructed for W7-X stellarator. X-ray pulse height analysis system was constructed. Determination of impurities concentration for selected discharges was calculated from measurements. Very good agreement was obtained between 
experimental and simulated spectra. The impurities concentrations were obtained with sufficient precision. This accuracy depends critically on the accuracy of electron temperature and density measurements. The collected measurement data and further calculations open opportunity to deliver input data for next level numerical analyses of transport in plasma, evolution of impurities concentration, effective gas puff and heating. There is also a possibility to determine $Z_{\text {eff }}$ with slight assumptions, including all contributions to radiation from plasma. The work is done in IFPiLM.

Joint European Torus - JET experiment is currently the largest facility for magnetic confinement plasma physics. It enables research and diagnostics on impurity identification by several different class cameras, and tracing the impurity behaviour during ${ }^{3} \mathrm{He}, \mathrm{Ne}$ and $\mathrm{D}$ gas injection. Mid $\mathrm{Z}$ and high $\mathrm{Z}$ impurities are of concern like: $\mathrm{Ni}, \mathrm{Fe}, \mathrm{Cu}, \mathrm{Mo}$, and $\mathrm{W}$. Impact of different gas injections on impurity behaviour is important for future development of energy generation tokamaks. It was observed that different plasma heating scenarios cause increased production of specific impurities. Increasing D gas injection rate and $\mathrm{He}$ concentration caused decrease of mid and high $\mathrm{Z}$ impurities concentrations.

Proton flux was measured using wide gap semiconductor detectors. The relevant detectors are: photoconductive single crystal diamond, single crystal SiC, interdigitated GaAs and fast InP. The detectors are blinded to visible and IR radiation which accompanies proton emission. In the experimental measurement set up the protons were generated with an titanium sapphire 10 TW fs laser. Single crystal SiC and diamond detectors are suitable to absolute measurements of proton flux. Medium band gap InP and GaAs detectors are useful of protons from laser plasma. InP interdigitated detectors assures 100-ps time resolution of measurements. Overloading effects are often observed at measurements of proton fluxes from laser plasma.

Protons were accelerated to high energies by ultra-intense fs laser pulses at IFPiLM photonic laboratory in cooperation with ELI. The main advantages of laser-driven ion accelerator are: up to $10 \mathrm{GV} / \mathrm{m}$ field strength, short ion acceleration path, compactness, high ion current density and ion beam intensity, very short fs-ps ion pulses. Major disadvantage is quite broad ion energy spectra. It has been found that for the laser intensities above $10^{22} \mathrm{~W} / \mathrm{cm}^{2}$ parameters of the generated proton beam weakly depend on the laser beam polarization and a dominant mechanism of proton/ion acceleration is RPA - radiation pressure. The obtained quasi monochromatic proton beam parameters are very good what can open the door for new areas of research in nuclear physics, high energy density physics or material research.

DAQ system was proposed for PANDA experiment at FAIR. The system has to cope with classical data congestion problem. Typical network architectures avoid packet dropping by creating backpressure on input port. This approach is based on assumption that traffic is distributed evenly over the network and sender has large enough buffer. PANDA DAQ role is to deliver all fragments of data generated by detectors during one epoch (duration of 2 us) to one reconstruction farm. Therefore traffic shape is vastly different and there are high spikes of data on egress port instead. Network implemented in this typical way is not suitable for PANDA DAQ because congestion on single egress port will corrupt data of following events, even though these events are not sent to congested port. ATCA hardware was used in the design. Front End Panda electronics monitors signals from detectors and in case of crossing a threshold data packet with timestamp is formed and sent to event building and filtering farm. There is no hardware triggering. All the data is processed and usefulness of event is estimated after reconstruction. DAQ system should provide way to deliver all fragments of the same farm. The work is done at KrUT and IF PAN in Kraków.

Pi-of-the-Sky experiment with data gathered for more than a decade participates in various research experiments supplementing the main astronomical science trends. The project is coordinated successfully by Mikołaj Kopernik Astronomical Research Centre. These include joining the network of dark matter search, changing stars cataloguing and betterment of data on them, looking for space debris orbiting on LEOs and participation in cataloguing, etc. Different algorithm are researched to process gathered data and prepare various choice and triggering mechanisms to reveal knowledge in big collection of data. One of the research directions is search for failed supernovae. Supernovae are stars with thermal runaway 1a, or core collapse all other cases. They are possible source of gravitational waves. Failed supernovae is a core-collapse star without EM counterpart. It should also produce gravitational wave but with no spectacular EM counterpart. Candidates are search among objects that were previously clearly visible and then disappeared. The gathered data after processing can put an upper bound on how often this process might appear. It is comparatively easy to recognize if some object has recently and unexpectedly appeared. Much different image recognition algorithms have to be used for unexpectedly disappearing objects. Database of previously well observed objects is used, supplemented with the list of observed objects from the current science runs. Comparisons are made of lists of objects observed during previous science runs and current science runs, and see if anything is missing. Clearly missing objects are investigated. The following issues have to be taken into consideration while processing images: data quality, not all nights have the same limiting magnitude, camera behaviour changes with time, some variable stars are dimming below limiting magnitude causing false positives, satellites could cause false negatives, large density of objects in galactic plane cause bad matching to catalogue.

Pi-of-the-Sky searches for variable objects/stars and uses multivariable analysis method MVA. Data analysis process bases on LUIZA framework. Variable objects include: supernovae, novae, flare stars, and variable stars. Astronomical image analysis with data reduction includes the following steps: image normalization - dark subtraction and flat correction, photometry - object identification and brightness estimate calculate Gauss PSF map and kernel, calculate signal using aperture based signal, astrometry - sky position determination, calibration - magnitudo scale determination from comparison to reference stars, and cataloguing results in the relevant database. Variable stars recognition uses the following assumptions for the algorithm, which has to be independent of the period and shape of the light curve. It has to be sensitive to small brightness changes around 0,1 magnitudo and should allow the background reduction from constant stars at the level minimum $95 \%$. Recognition of variable stars bases on the following assumptions. $1 \%$ of stars in the Sky are variable. Sky monitoring should be done with high temporal resolution where each star is 
found in many frames. Measurements of the brightness of constant stars should form a normal distribution. Learning algorithm and MVA analysis is applied because one does not know the functional dependencies between variables, and one assumes that there are no predictions. Many variables are used because one wants to make optimum use of all available information. The distribution of likelihood of multiple variables mostly include more information than the sum of distributions of individual variables. The goal is to separate signal and background. The learning algorithm divides the data set to two parts - one used for training and the second for testing. The following variables are used; RMS, Kurtosis, Asymmetry and Skewness. The input for the analysis are brightness distribution parameters and the output is the probability that the distribution of magnitude presents a variable star, assuming that the background is rejected with high signal efficiency. Period was estimated for selected stars. It was shown that the analysis of the higher statistical moments like kurtosis asymmetry and skewness can be helpful to recognition of variable stars.

Pi-of-the-Sky, a search for short optical transients, participates in LSC-Virgo EM follow-up project. New data analysis framework was organized called LUIZA with better brightness estimation and subpixel position estimation, using methods taken from HEP experiments. EM follow-up of gravitational waves GW triggers would be carried out by LSCVirgo and astronomical partners. After four successful detections of gravitational waves, all triggers are going to be publicly available. GLADE reference catalogue is used instead of GWGC.

Pi-of-the-Sky participated in detection of GRB160625B. It was very bright burst, very well monitored in muti-wavelegth spectra. Three dramatically different isolated sub-bursts separated by long quiescent background were observed with different spectral properties dominated by distinct physical processes: $0,8 \mathrm{~s}-180 \mathrm{~s}$ silence-35s, 339s silence, 212s. For the first time thermal and non-thermal radiation was observed in a single GRB. The first sub burst originates from cocoon emission surrounding the relativistic jet. The second sub-burst originates from photosphere emission and internal shock relativistic jet. The third sub-burst originates from internal and external shocks relativistic jet. First sub-burst shows initial iron collapse that forms a black hole or a rapidly spinning magnetar surrounded by some high angular momentum materials. The initial hyperaccretion onto the central object forms a matter-dominated fireball via neutrino-anti-neutrino annihilations. The first several pulses within the ejecta merge and eventually break out of the star to make a successful thermal-dominant GRB precursor. Central engine is strongly magnetized when the second jet is launched, so that the emission would be characterized by a synchrotron spectrum without a significant thermal component from the photosphere. The central engine would reactivate again similar to the typical long GRBs that show significant late time X-ray flares. Energy emitted during the first and second episode power the third emission episode. Second successful GRB observation by the Pi-of-the-Sky has confirmed the usefulness of the project and effectiveness of its observation strategy. Pi-of-the-Sky does routine wide lens surveys, high time resolution, and automatic flash detection, of all sky during every night, participates in LSC-Virgo follow up project, collects data on changing stars and space debris on
LEOs, and uses its database for confinement calculations on different astronomical effects.

AGH electronics laboratory specializes in building and experimenting with readout systems for X-ray pixel detectors for research experiments in neurobiology and physics. Large amount of data has to be read from the chip. One of the researched solutions is scalable low voltage signalling SLVS transceiver/driver working at least with 4 Gbps readout rates.

\section{CONCLUSIONS, WILGA 2017, WILGA 2018, AND FuturE WILGA MEETINGS}

One of WILGA 2017 Sessions was devoted to teaching of photonics, Internet and computer engineering. Modern computers with advanced microprocessors, due to performance enhancement mechanisms, cannot be reasonably monitored, and it is not possible to show the process of instruction execution to the students. Students have problems understanding the basics of computer hardware operation, including the process of instruction execution like opcode, fetch, argument read, result write, and other fundamental concepts like stack operation, addressing modes, etc. All these operations may be shown directly only using real hardware from eighties. Software simulators are virtual not hardware. At II WUT there was constructed a real working computer based on classical CPU but with extended hardware monitoring capabilities. Similar introductory laboratories in computer engineering, photonics and communications were developed at other universities.

XL edition of WILGA 2017 Symposium gathered a record number of participants young researchers active in photonics and electronics. The work presented during Wilga 2017 showed the state-of-art of these fast developing research and application fields in Poland, as developed in university laboratories by young scientists. The researched subjects are very current, attractive and competitive. A lot of subjects is centred around the wide area and different aspects of the Internet of Things.

Started two decades ago WILGA Symposium evolutionarily grew from a small local event to a large national meeting of young scientists with considerable international participation. The organizers did not expect such evolution. The number of participants increased slowly, showing some demand for such a broader meeting mixing young researchers now for nearly all technical universities from Poland. Wilga is diligently addressing this demand for keeping the annual large meeting of young researchers working in advanced electronics and photonics systems. The organizers hope that the meeting serves well the research and academia communities.

The next Symposium 2018 WILGA Applications of Photonics and Internet Engineering will be traditionally organized in two editions the $41^{\text {st }}$ and $42^{\text {nd }}$, Winter $25-28$ January 2018 and Summer 26 May - 03 June 2018. The organizers of the Symposium WILGA 2017 invite young scholars and their mentors to participate [http://wilga.ise.pw.edu.pl]. So far Wilga seems to well serve the young community of researchers in the fields of advanced electronics and photonics. The style and conditions of this flexible meeting, essentially with no stiff rules, is expected to fit dynamically to the style and needs of ever changing young science. The survivability and positive future of WILGA depends strongly on this adaptability strength to the fast changing conditions of doing demanding research by young 
scientists. Missing this relatively narrow target may result very fast in declined interest in this type of large, multidisciplinary, yet exceptional meeting. One of the choices for active tracking the right target and its movements is to make the young researchers do such meeting for their colleagues. This costs a lot of effort for them but hopefully finally pays off.

\section{REFERENCES}

[1] J.W.Modelski, R.S.Romaniuk, Committee of Electronics and Telecommunications, Polish Academy of Sciences, Structure Activities - Perspectives, International Journal of Electronics and Telecommunications, vol.61, no.1, 2015, pp. 49-56

[2] R.S.Romaniuk, WILGA Symposium on Photonics Applications, Photonics Letters of Poland, vol.1, no.2, 2009, pp.46-48,

[3] R.S.Romaniuk, Wilga photonics and web engineering 2010, Photonics Letters of Poland 2 (2), 2010, pp.55-57

[4] R.S.Romaniuk, Electronic and Photonic Systems WILGA 2014, International Journal of Electronics and Telecommunications, vol.60, no.3, 2014, pp.271-276

[5] R.S.Romaniuk, Photonics and web engineering - WILGA Summer 2016, Proc.SPIE, vol. 10031, 2016, art.no.1003103

[6] R.S.Romaniuk, Photonics applications and web engineering WILGA 2017, Proc. SPIE, vol.10445, 2017, art.no.1044501

[7] R.S.Romaniuk, Multicore optical fibres, Revue Roumaine de Physique 32(1-2), 1987, pp.99-112

[8] A.Dybko, W.Wróblewski, J.Maciejewski, et al., Fiber optic probe for monitoring of drinking water, Proc.SPIE, vol.3105, 1997, pp.361-366

[9] R.Romaniuk, J.Dorosz, Measurement techniques of tailored optical fibres, Proc.SPIE, vol.5064, 2003, pp.210-221

[10] R.S.Romaniuk, Optical fiber transmission with wavelength multiplexing - Faster or denser?, Proc.SPIE, vol. 5484, 2004 , pp.19-28
[11] A.Burd, M.Ćwiok, H.Czyrkowski, et al., Pi of the sky - automated search for fast optical transients over the whole sky, Astronomische Nachrichten, 325 (6-8), 2004, pp.674

[12] T.Czarski, R.Romaniuk, K.Pozniak, S.Simrock, Cavity control system advanced modeling and simulations for TESLA linear accelerator and free electron laser, Proc.SPIE, vol.5484, 2004, pp.69-87

[13] T.Czarski, K.Poźniak, R.Romaniuk, S.Simrock, TESLA cavity modelling and digital implementation, Proc.SPIE, vol.5484, 2004, pp.111-129

[14] K.T.Pozniak, T.Czarski, R.S.Romaniuk, Functional analysis of DSP blocks in FPGA chips for application in TESLA LLRF system, Proc. SPIE vol. 5484, 2004, pp.130-138

[15] W.M.Zabolotny, P.Roszkowski, K.Kierzkowski, et al., Distributed embedded PC based control and data acquisition system for TESLA cavity controller and simulator, Proc.SPIE, vol.5448, 2004, pp.171-179

[16] K.T.Pozniak, T.Czarski, R.S.Romaniuk, FPGA based cavity simulator and controller for TESLA Test Facility, Proc.SPIE, vol.5775, 2005, pp.9-21

[17] P.Pucyk, T.Jeżyński, W.Koprek, et al., DOOCS server and client application for FPGA based TESLA cavity controller and simulator, Proc.SPIE, vol.5775, 2005, pp.52-60

[18] S.Chatrchyan, V.Khachatryan, A.M.Sirunyan, et al., Performance and operation of the CMS electromagnetic calorimeter, JINST 5 (3), 2010, art.no.T03010

[19] R.S.Romaniuk, Modal structure design in refractive capillary optical fibres, Photonics Letters of Poland, 2010, 2 (1), pp.22-24

[20] R.S.Romaniuk, Geometry design in refractive capillary optical fibers, Photonics Letters of Poland, 2 (2), 2010, pp.64-66

[21] R.S.Romaniuk, J.Dorosz, W.Wójcik, P.Mergo, R.Buczyński, Optical fiber technology in Poland - four decades of development 1975-2015, Proc.SPIE 9816, 2015, art.no.981603. 